\title{
Stability of Colloidal Silver Nonoparticle Solutions Prepared by Chemical Reduction
}

\author{
H.M. Al-Khateeb*, F.Y. Alzoubi, M.K. Alqadi And M.A. Mohammed \\ Department of Physics, Jordan University of Science and Technology (JUST), P.O. Box 3030, Irbid 22110, Jordan \\ Three different samples of silver colloidal nanoparticles were synthesized via the chemical reduction method \\ using three different stabilizers (citrate, PVP, SDS). The prepared samples were characterized using UV-vis spec- \\ tra, TEM imaging and XRD analysis. Our results show that absorption spectra of the three samples were stable \\ over time. Slight changes have occurred in the spectrum of the PVP sample, indicating lesser stability of this \\ sample. TEM images show that silver nanoparticles are well dispersed in the three samples and the sizes of these \\ nanoparticles range from $10.1 \pm 4.2 \mathrm{~nm}$ to $13.5 \pm 4.7 \mathrm{~nm}$. XRD curves show that the prepared silver nanoparticles \\ are of FCC crystal structure. The XRD curves were used to calculate the size of our nanocrystals and it is found \\ that the average size of the nanoparticles in the citrate sample is $19.9 \mathrm{~nm}$, in the PVP sample it is $11.5 \mathrm{~nm}$, and \\ in the SDS sample it is $12.5 \mathrm{~nm}$. In conclusion, the prepared samples were stable over time and no considerable \\ aggregation was observed.
}

DOI: 10.12693/APhysPolA.134.217

PACS/topics: silver colloidal nanoparticles, chemical reduction method, sodium citrate, polyvinylpyrrolidone, sodium dodecyl sulfate

\section{Introduction}

Recently, metallic nanoparticles are being widely used in different areas of science and technology due to their unique electronic, optical, mechanical, magnetic and chemical properties [1]. These special and unique properties are attributed to their high surface to volume ratio $[2,3]$.

There are two approaches of synthesis of nanoparticles: the bottom-up and the top-to-bottom approaches $[4,5]$. Top-to-bottom approach is a separation method by which an external force is applied to a solid (bulk) that leads to its fragmentation into smaller particles. Ball milling can be given as an example of such approach. Bottom up approach is to form the nanoparticles from atoms of gas or liquid, based on atomic transformation or molecular condensations.

In this study, we will follow the bottom up approach to prepare silver nanoparticles via the chemical reduction method in which reducing agent is provided to neutralize silver ions. There are a variety of reducing agents to be used such as sodium citrate, ascorbate, poly(ethyleneglycol) and ribose. After neutralization, silver atoms start to agglomerate to form silver nanoparticles within the suspension.

To prevent aggregation, a stabilizer such as sodium citrate, polyvinylpyrrolidone (PVP), and sodium dodecyl sulfate (SDS) has to be added within the preparation $[6-10]$. The main aim of our study is to discuss the effect of three stabilizers, used as the protective agents.

UV-visible spectrophotometer is used to obtain absorption spectra of the prepared colloidal suspension of silver

*corresponding author; e-mail: hkhateeb@just.edu.jo nanoparticles. Silver nanoparticles are characterized by the surface plasmon resonance (SPR) at $420 \mathrm{~nm}$. The absorption peak at this wavelength can be used to get information about the size and shape of the nanoparticle as well as the information about the refractive index and subsequently information about the dielectric properties. Transmission electron microscopy (TEM) is used to measure average sizes and size distribution of nanoparticles. X-ray diffraction (XRD) patterns are obtained to study the crystalline structure of the prepared colloidal nanoparticles as well as to calculate their size according to Scherrer equation [11-13].

\section{Materials and methods}

There are various methods for preparing nanoparticles. These methods differ in cost, difficulty, tools and setup. One of the most famous methods is chemical reduction method which is simple as well as inexpensive [14]. Three different samples of colloidal silver nanoparticles were prepared. The samples differ according to the type of the stabilizer used in the synthesis process. These samples are:

1. Citrate sample was prepared using trisodium citrate as the stability agent. During preparation $0.01056 \mathrm{~g}$ of ascorbic acid (purity 99\%) and $0.08816 \mathrm{~g}$ of trisodium citrate were dissolved in $100 \mathrm{ml}$ of deionized water. Then, the solution was left on a hot plate under continuous stirring and condensation to reach the boiling point of $90^{\circ} \mathrm{C}$. After that $1 \mathrm{ml}$ of silver nitrate $\left(\mathrm{AgNO}_{3}\right)$ was added drop by drop until the color of the solution changed from colorless to yellow within 5-10 minutes. The solution was left boiling for one hour with stirring to obtain the nanoparticles that were kept later in a dark medium. 
2. PVP sample was prepared using polyvinyl pyrrolidone (PVP) as the stability agent. During preparation $2.55 \mathrm{~g}$ of (PVP) and $2.55 \mathrm{~g}$ of silver nitrate were dissolved in $50 \mathrm{ml}$ of deionized water. The suspension was stirred at room temperature for two hours [15].

3. SDS sample was prepared using sodium dodecyl sulfate (SDS) as the stability agent. During preparation $0.425 \mathrm{~g}$ of $\mathrm{AgNO}_{3}$ were dissolved in $500 \mathrm{ml}$ of deionized water, and then $1 \mathrm{~g}$ of ribose and $0.5 \mathrm{~g}$ of SDS were added to the solution. The suspension was stirred and heated up to $50^{\circ} \mathrm{C}$ and then left for 30 minutes [5].

Various characterization techniques were employed in this study to verify the formation of the colloidal nanoparticles as well as to investigate their stability. UVvisible spectrophotometer was used to obtain the absorption spectra of the prepared samples which are considered as the first step in detecting the formation of the colloidal silver nanoparticles by observing their surface plasmon peak at $420 \mathrm{~nm}$. Moreover, these spectra were used to monitor the stability of the colloidal solutions by detecting changes occurring to these spectra. XRD patterns were obtained to investigate the crystalline structure of the prepared colloidal silver nanoparticles as well as to estimate the size of these nanoparticles. In addition, TEM images were used to study the stability and size of the prepared samples.

\section{Results and discussion}

In this section we present and discuss the results for the prepared samples of colloidal silver nanoparticles. The color of each sample after stirring was examined with naked eye. The color gives a clear impression about the formation of the silver nanoparticles (see Fig. 1).
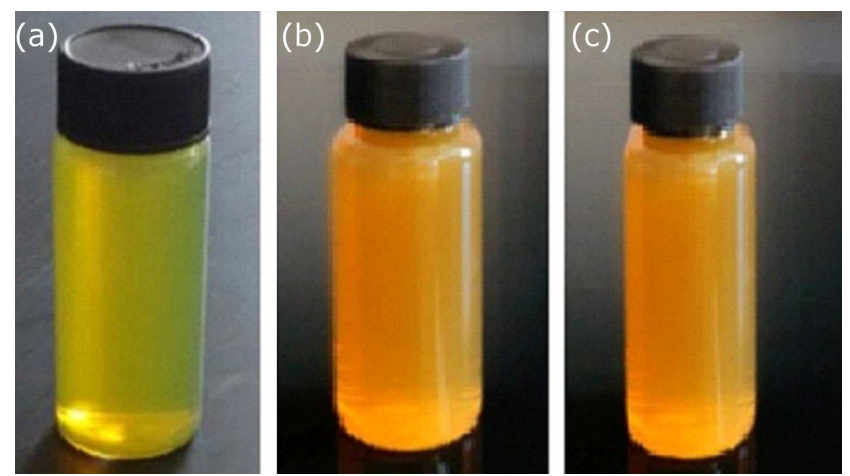

Fig. 1. The color of the prepared samples: (a) the citrate sample, (b) the PVP sample and (c) the SDS sample.

UV-visible absorption analysis in the range of 300 $700 \mathrm{~nm}$ was performed to detect the formation of silver nanoparticles in the prepared samples. SPR peak was observed around $420 \mathrm{~nm}$ in all prepared samples. This peak is a characteristic property of silver nanoparticles. Figure 2 shows the typical absorption spectra for the three prepared samples of colloidal silver nanoparticles. For citrate sample it is clear that there is a surface plasmon absorption band centered at a wavelength of $420 \mathrm{~nm}$. The peak is very strong and sharp, indicating the uniformity of size and shape of the prepared colloidal silver nanoparticles. This peak is a characteristic property of $\mathrm{Ag}$ colloidal nanoparticles and is considered as one of the pointers of the formations of nanoparticles.

For PVP sample, Fig. 2b shows that the absorption peak is not as sharp as that of the citrate sample, indicating a lesser uniformity of sizes of nanoparticles contained in the suspension. The maximum absorption occurs at $427 \mathrm{~nm}$. The absorption spectrum of the SDS sample, as shown in Fig. 2c, has a resonance peak at the wavelength of $412 \mathrm{~nm}$ and is similar in sharpness to that of the citrate sample while being sharper than taht of the PVP sample.

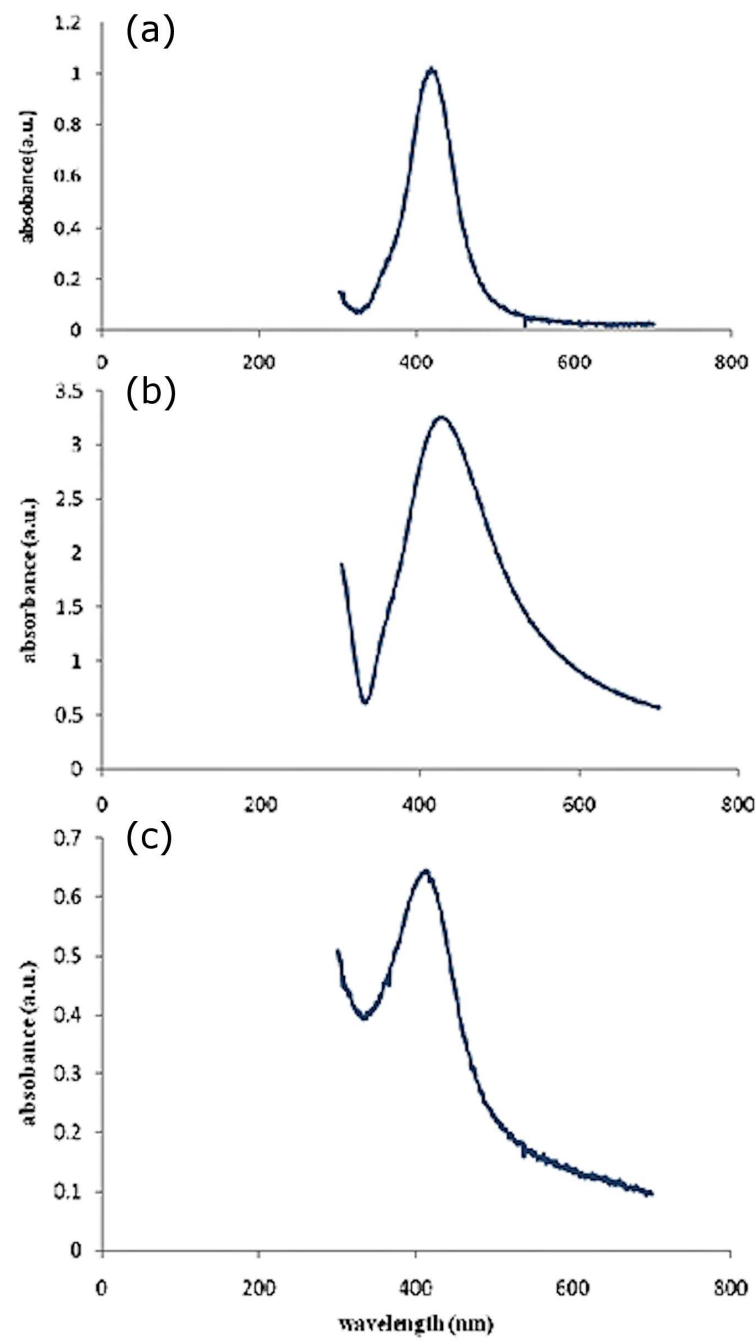

Fig. 2. UV-vis spectra for citrate (a), PVP (b) and SDS (c) samples after preparation. 

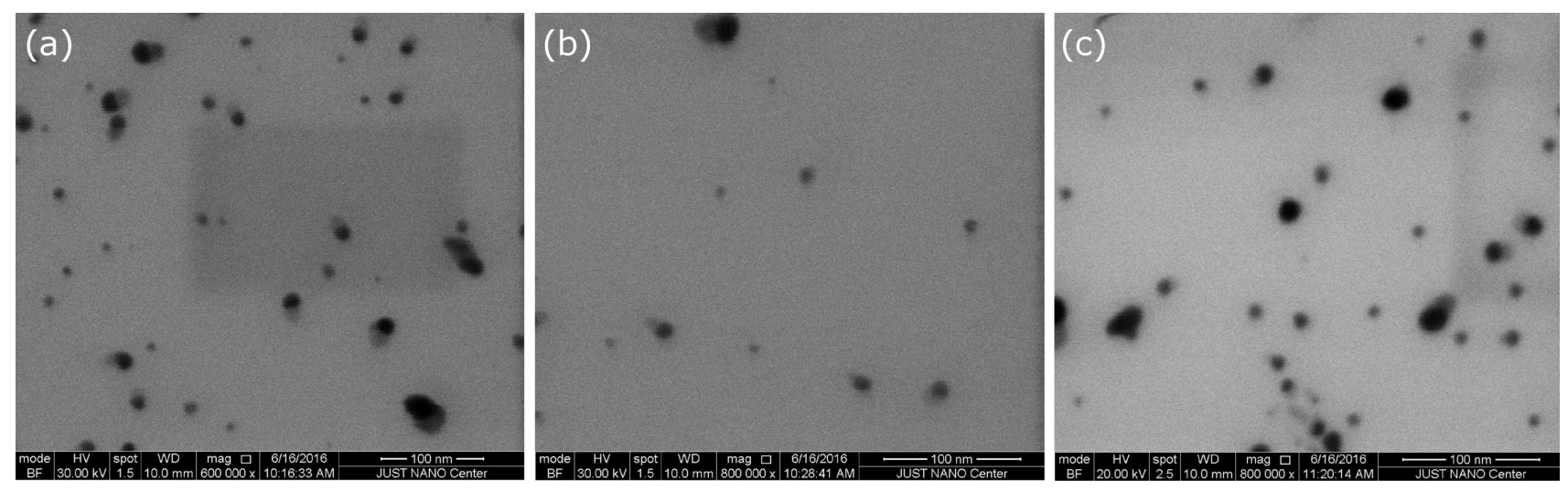

Fig. 3. TEM images for citrate sample (a), PVP sample (b), and SDS sample (c) after preparation.
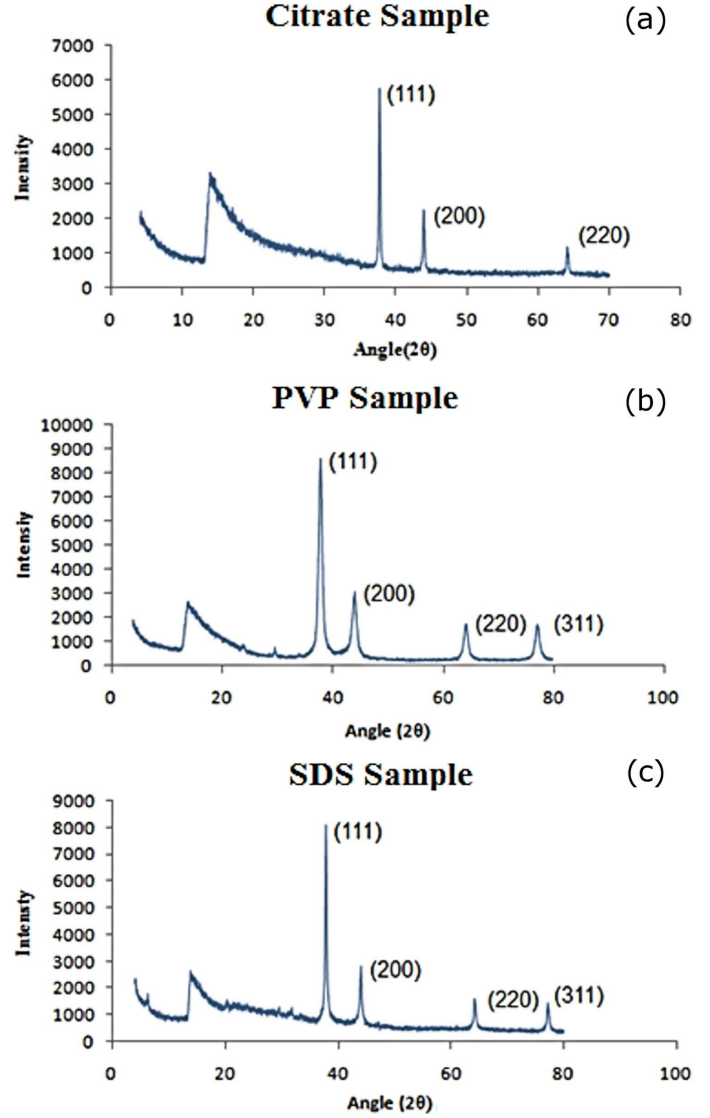

Fig. 4. X-ray diffraction spectra for citrate (a), PVP (b) and SDS (c) samples.

Figure 3 shows the three TEM images of the three samples prepared using different stabilizers. One may observe that the three colloidal samples are stable since no aggregation is observed and the nanoparticles are well dispersed in the suspension. The shapes of the nanoparticles in all three samples are mainly spherical. The TEM images can be used to estimate the sizes of the nanoparticles in each sample. The sizes of nanoparticles are $13.5 \pm 4.7 \mathrm{~nm}, 11.9 \pm 4.3 \mathrm{~nm}$ and $10.0 \pm 4.2 \mathrm{~nm}$ in samples prepared using citrate, PVP and SDS, respectively.
XRD patterns are investigated to figure out the structure of our prepared silver nanoparticles. It is found that all silver nanoparticles are of FCC structure, in agreement with previous studies [4, 18]. Figure 4 displays the XRD curves of the three prepared samples. It is clear that the peaks are located at $2 \theta=37.68^{\circ}, 43.92^{\circ}$ and $64.08^{\circ}$ for citrate sample (Fig. $4 \mathrm{a}$ ), $2 \theta=37.89^{\circ}$, $44.18^{\circ}, 64.38^{\circ}$ and $77.2^{\circ}$ for PVP sample (Fig. 4b), and $2 \theta=37.84^{\circ}, 44.06^{\circ}, 64.24^{\circ}$ and $77.18^{\circ}$ for SDS sample (Fig. 4c), which correspond to the planes (111), (200), (220) and (311) respectively.

The XRD data can be used to calculate the average size of nanocrystals according to Scherrer equation: $D=0.94 \lambda / \beta \cos \theta$, where $D$ is the diameter of the nanoparticle, $\lambda$ is the wavelength and $\beta$ is the full width at half maximum. Table I lists the averages sizes obtained using the XRD spectra as well as using the TEM images. It is clear that the results obtained using the XRD are almost comparable with those obtained from the TEM images. Our calculated sizes are in good agreement with the calculated values from the literature [15-17].

The average sizes of silver nanoparticles

TABLE I prepared in this study.

\begin{tabular}{c|c|c}
\hline \hline Sample & TEM imaging $[\mathrm{nm}]$ & XRD curves $[\mathrm{nm}]$ \\
\hline Citrate & $13.5 \pm 4.7$ & 19.9 \\
PVP & $11.7 \pm 4.7$ & 11.5 \\
SDS & $10.1 \pm 4.2$ & 12.53
\end{tabular}

For example, Mikac et al. [15] obtained silver nanoparticles with sizes of $16.7 \pm 4.4$ and $12.6 \pm 4.2 \mathrm{~nm}$ using citrate and PVP as stabilizers, respectively. TEM images were used to measure the sizes of nanoparticles. Stable silver nanoparticles with sizes of $12.5 \pm 4.9 \mathrm{~nm}$ have been obtained by Mallmann et al. [16] using ribose as a reducing agent and SDS as a stabilizer. In a study done by Lanje et al. [17], silver nanoparticles were prepared by reducing silver nitrate $\left(\mathrm{AgNO}_{3}\right)$ with glucose in the presence of PVP as the protective agent. TEM images showed that the average particle size of these nanoparticles was about $15 \mathrm{~nm}$. 

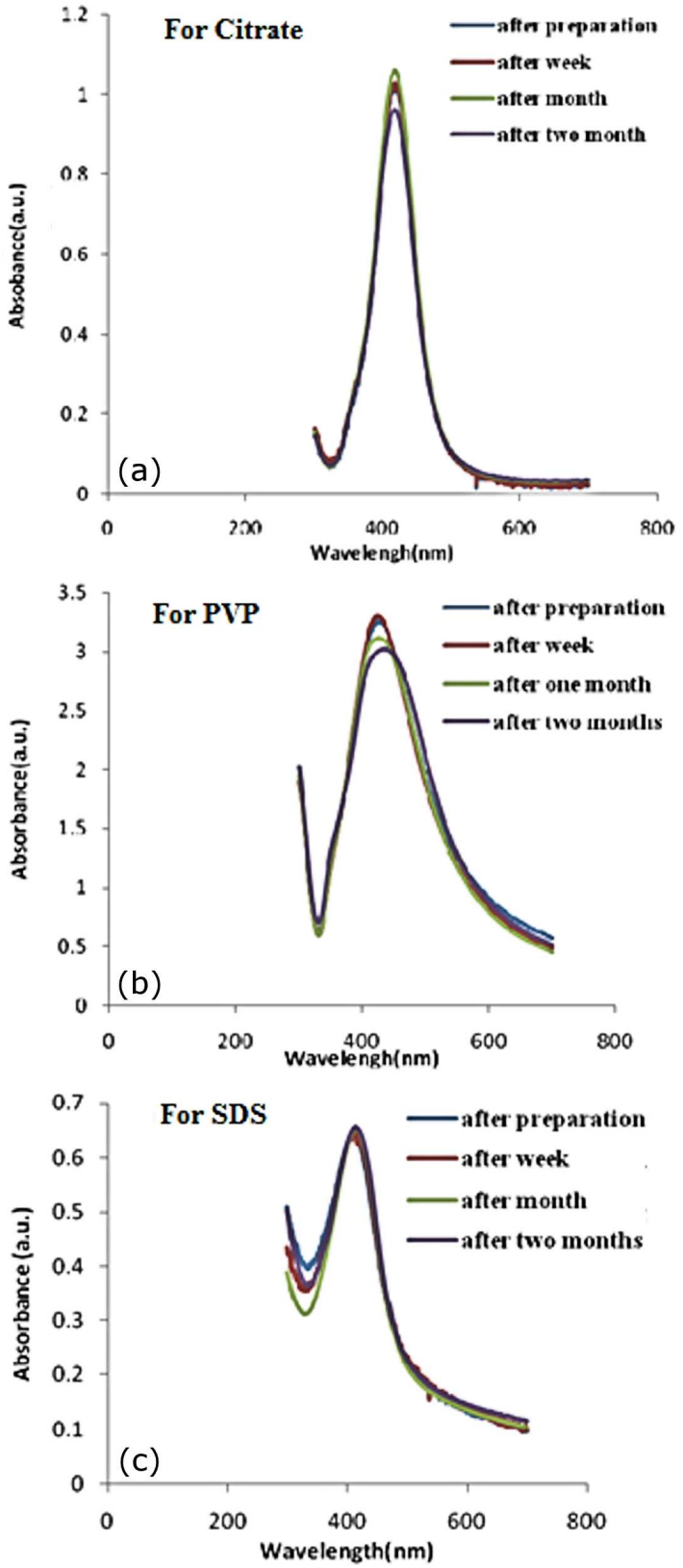

Fig. 5. Stability over the time of the samples prepared using various stabilizers.

One of the challenges facing researchers who are interested in preparing colloidal suspensions of nanoparticles is the stability of their samples. In this study, we prepared three samples using different stabilizers: sodium citrate was used as representative of electrostatic stabilizers, while PVP and SDS were used as representatives of steric stabilizers.

The stability of the prepared samples was monitored by observing any changes that occur to the absorption peaks over time. Figure 5 presents the absorption spectra of colloidal silver nanoparticles prepared using three different stabilizers. For sodium citrate sample (Fig. 5a) there were no considerable changes in absorption spectra that were taken over a period of two months. This indicates that the silver nanoparticles are stable.

For PVP stabilizer (Fig. 5b), there are slight changes to the shape of the absorption peak. These changes include a reduction in the absorption at the resonance frequency, accompanied by a little shift towards the long wavelength region. This usually indicates to an increase in the size of the particles, as well as a little aggregation could be occurring. We may conclude that the PVP sample is less stable than the citrate sample. This difference may be attributed to some technical problems in keeping the sample in a cool environment. It is worth mentioning that the PVP sample is prepared at room temperature while the other two samples were prepared at $90^{\circ} \mathrm{C}$ for citrate sample and $50^{\circ} \mathrm{C}$ for the SDS sample. For SDS as the stabilizer (Fig. $5 \mathrm{c}$ ) it is clear that no considerable change had occurred to absorption peak over two months. Mallmann et al. [16] prepared a sample using SDS as the stabilizer and they noticed that their sample was stable up to four months when stored at room temperature and exposed to ambient light.

\section{Conclusions}

Three different samples were prepared using different stabilizers (sodium citrate, PVP, and SDS). The chemical reduction method was used in preparing the three samples. The prepared samples were characterized using their absorption spectra, TEM images and XRD spectra. The absorption spectra obtained over a period of two months indicate stable colloidal suspensions since no remarkable change occurred to the surface plasmon peaks over this period, except that a slight change has occurred in the case of the PVP sample.

The TEM images support our analysis of absorption spectra since all images show well dispersed silver nanoparticles and no aggregation in the form of clusters was observed. Moreover, these images were used to estimate the sizes of silver nanoparticles. It was found that the average size of particles the citrate sample is $13.5 \pm 4.7 \mathrm{~nm}$, in the PVP sample it is $11.9 \pm 4.3 \mathrm{~nm}$ and for the SDS sample it is $10.1 \pm 4.2 \mathrm{~nm}$.

The XRD curves show the crystalline structure of the prepared silver colloidal nanoparticles. It is found that the patterns have peaks at $2 \theta=37.68^{\circ}, 43.92^{\circ}, 64.08^{\circ}$ for citrate sample, $2 \theta=37.89^{\circ}, 44.18^{\circ}, 64.38^{\circ}, 77.2^{\circ}$ for PVP sample and $2 \theta=37.84^{\circ}, 44.6^{\circ}, 64.24^{\circ}, 77.18^{\circ}$ for SDS sample, corresponding to planes of (111), (200), (220) and (311), respectively. Furthermore, these curves were used to calculate the size of the nanoparticles in each sample using Scherrer equation. It is found that the sizes of the nanoparticles are $19.9 \mathrm{~nm}, 11.5 \mathrm{~nm}$, and $12.53 \mathrm{~nm}$ in the citrate, PVP, and SDS samples, respectively.

In general, the sizes of nanoparticles calculated using XRD curves are in agreement with the sizes obtained from the TEM images. In conclusion, our three prepared samples are stable over a period of at least two months and we expect them to be stable for a longer period of time. 


\section{Acknowledgments}

The authors would like to thank Jordan University of Science and Technology (JUST) for the support provided by the Deanship of Scientific Research. They would also like to thank Prof. B. Albiss and Prof. M.-Ali Al-Akhras for their help in using the facilities of Nanotechnology Institute and the Lab. of Biomedical Physics at JUST.

\section{References}

[1] D. Myers, Surfaces, Interfaces, and Colloids: Principles and Applications, Wiley, New York 1999.

[2] C. Santos, R. Oliveira, M. Seckler, 18th International Symposium on Industrial Crystallization, Zurich 2011

[3] S. Lal Pal, U. Jana, P.K. Manna, G.P. Mohanta, R. Manavalan, J. Appl. Pharmac. Sci. 1, 228 (2011).

[4] M. Forough, K. Fahadi, Turkish J. Eng. Env. Sci. 34, 281 (2011).

[5] P.S. Mdluli, Ph.D. Thesis, University of Zululand, South Africa 2009.

[6] R. Usman, Ph.D. Thesis, Kagoshima University, Japan 2015

[7] J. Widoniak, S. Eiden-Assmann, G. Maret, Colloids Surf. A: Physicochem. Engin. Asp. 270, 340 (2005).
[8] A.A. El-Kheshen, S.F. Gad El-Rab, Der. Pharma. Chem. 4, 53 (2012).

[9] D. Malina, A. Sobczak-Kupiec, Z. Wzorek, Z. Kowalski, Digest J. Nanomat. Biostruct. 7, 1527 (2012).

[10] Y. Yin, Z. Li, Z. Zhong, B. Gates, Y. Xia, S. Venkateswaran, J. Mater. Chem. 12, 522 (2002).

[11] V. Amendola, Ph.D. Thesis, University of Padova, Padova, Italy 2008.

[12] T. Panigrahi, Master Thesis, National Institute of Technology, Rourkela, India 2013.

[13] A. Panacek, L. Kvitek, R. Prucek, M. Kolar, R. Vecerova, N. Pizurova, V.K. Sharma, T. Nevecna, R. Zboril, J. Phys. Chem. B 110, 16248 (2006).

[14] P.C. Lee, D. Meisel, J. Phys. Chem. 86, 3391 (1982).

[15] L. Mikac, M. Ivanda, M. Gotić, T. Mihelj, L. Horvat, J. Nanoparticle Research 16, 2748 (2014).

[16] E.J. Mallmann, F.A. Cunha, B.N. Castro, A.M. Maciel, E.A. Menezes, P.B. Fechine, Rev. Inst. Med. Trop. São Paulo 57, 165 (2015).

[17] A.S. Lanje, S.J. Sharma, R.B. Pode, J. Chem. Pharm. Res. 2, 478 (2010).

[18] S.K. Brar, M. Verma, TrAC Trends in Analyt. Chem. 30, 4 (2011). 